\title{
Diagnóstico Lean Six Sigma en el proceso de impresión offset. Un aporte a la productividad de las pequeñas empresas del sector industrial por parte de estudiantes de ingeniería
}

Javier Neira-Rueda ${ }^{1}$

Corporación Universitaria Minuto de Dios javier.neira@uniminuto.edu

Alejandra Rojas ${ }^{2}$

Corporación Universitaria Minuto de Dios projasgalvi@uniminuto.edu.co

Luis Eduardo Díaz ${ }^{3}$

Corporación Universitaria Minuto de Dios Idiazmaldon@uniminuto.edu.co

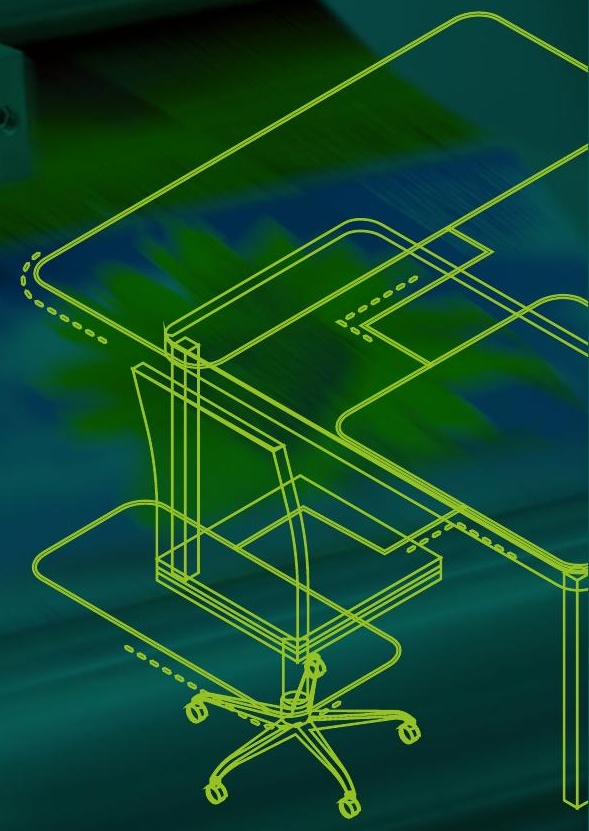

Fecha de aprobación: 19 de diciembre de 2019

Cómo citar este artículo: Neira-Rueda, J.; Rojas, A.; Díaz, L. E. (2019). Diagnóstico Lean Six Sigma en el proceso de impresión offset. Un aporte a la productividad de las pequeñas empresas del sector industrial por parte de estudiantes de ingeniería. Revista Ontare, 7, 49-70.

DOI: https://doi.org/10.21158/23823399.v7.n0.2019.2556

\footnotetext{
${ }^{1}$ Ingeniero Textil y Papelero de la Universitat Politéccnica de Valencia. Master Ingeniería de Análisis de Datos, Mejora de Procesos y Toma de decisiones de la Universitat Politécnica de Valencia. Doctorado en Estadística y optimización de la Universitat Politécnica de Valencia. ORCID: https://orcid.org/0000-0003-1074-4372

${ }^{2}$ Ingeniera industrial de la Corporación Universitaria Minuto de Dios y Auditora Interno en Sistemas de Gestión Integrados HSEQ - ISO 9001:2015, ISO 14001:2015 e ISO 45001:2018. ORCID: https://orcid.org/0000-0002-8317-2400

${ }^{3}$ Ingeniero industrial de la Corporación Universitaria Minuto de Dios. ORCID: https://orcid.org/0000-0001-5172-0065
} 


\section{RESUMEN}

Este documento estructura y propone los pasos para mejorar los procesos internos de impresión en la compañía Gráficas Jaiber Ltda., dedicada a la impresión offset y especializada en la producción de cajas plegadizas y etiquetas desde 1976. El artículo se enfoca en la mejora de los índices de calidad en impresión offset de la compañía desde la metodología Lean Six Sigma y tiene como objetivo principal aplicar un modelo de mejora continua que permita aumentar la productividad; esto mediante la reducción de la variabilidad en los procesos para garantizar así mayor calidad. En el propósito de mejorar este proceso se recolectó y analizó información, y se recomiendan acciones de control y mejora. Se emplearon herramientas de diagnóstico tales como las $5 \mathrm{w}$-las cinco preguntas-, Pareto, y diagrama causa y efecto, que luego de ser analizadas, permitieron encontrar las causas asignables de las problemáticas en los procesos de impresión offset. Finalmente, el análisis permitió a los ingenieros industriales de la Uniminuto, quienes buscan y tienen como filosofía institucional mejorar la productividad de las pequeñas y medianas empresas, otorgar recomendaciones dirigidas a la mejora continua con la metodología DMAIC, mediante acciones que me impacten de manera positiva los procesos internos y la productividad de esta empresa colombiana.

Palabras clave: Lean Six Sigma, investigación operativa; gestión de la calidad; mejora de la calidad; industria de impresión; procesos de producción. 


\section{Lean Six Sigma diagnostics in the offset printing process: A contribution from engineering students to the productivity of small companies in the industrial sector}

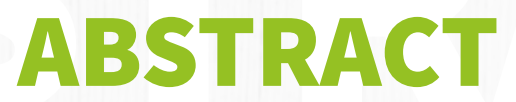

This document structures and proposes the steps to improve the internal printing processes at Graficas Jaiber Ltda., a company that is dedicated to offset printing and that is specialized in the production of folding carton boxes and labels since 1976. The article focuses on the improvement of the company's offset printing quality indexes from the Lean Six Sigma methodology, with the main objective of applying a model for continuous improvement that allows increasing productivity by reducing the variability in the processes so that greater quality can be guaranteed. Pursuant to improve this process, information was collected and analyzed, and control and improvement actions were recommended. Diagnostic tools such as the $5 \mathrm{w}$ (the five questions), Pareto, and cause and effect diagram were used, which after being analyzed, allowed to find the assignable causes of the problems in the offset printing processes. Finally, the analysis allowed the industrial engineers from Uniminuto, who seek and have the improvement of productivity in small and medium enterprises as an institutional philosophy, to provide recommendations aimed at the continuous improvement with the DMAIC methodology; through actions that impact positively the internal processes and productivity of this Colombian company.

Keywords: Lean Six Sigma; operational research; quality management; quality improvement; printing industry; production processes. 


\section{Lean diagnóstico Six Sigma no processo de} impressão offset. Uma contribuição para a

\section{produtividade de pequenas empresas do setor industrial por estudantes de engenharia}

\section{RESUMO}

Este documento estrutura e propõe as etapas para melhorar os processos internos de impressão na empresa Graficas Jaiber Ltda., dedicada à impressão offset e especializada na produção de caixas dobráveis e etiquetas desde 1976. O artigo se concentra na melhoria dos índices da qualidade em impressão offset da empresa a partir da metodologia Lean Six Sigma e seu principal objetivo é aplicar um modelo de melhoria contínua que aumenta a produtividade; isso reduzindo a variabilidade nos processos para garantir maior qualidade. Para melhorar esse processo, as informações foram coletadas e analisadas, e ações de controle e melhoria são recomendadas. Foram utilizadas ferramentas de diagnóstico como o $5 \mathrm{w}$ - as cinco perguntas - Pareto, e o diagrama de causa e efeito, que, depois de analisados, permitiram encontrar as causas atribuíveis dos problemas nos processos de impressão offset. Por fim, a análise permitiu aos engenheiros industriais da Uniminuto, que buscam e têm a filosofia institucional de melhorar a produtividade de pequenas e médias empresas, fazer recomendações visando à melhoria contínua com a metodologia DMAIC; através de ações que impactam positivamente os processos internos e a produtividade dessa empresa colombiana.

Palavras-chave: Lean Six Sigma, investigação operativa, gestão de qualidade, melhoria de qualidade, indústria de impressão, processos de produção. 


\section{Diagnostic Lean Six Sigma dans le processus d'impression offset: une contribution des étudiants d'ingénieurie de l'université Uniminuto à la productivité des petites entreprises du secteur industriel}

\section{RÉSUMÉ}

Ce document structure et propose des étapes d'amélioration des processus d'impression offset de l'imprimerie Graficas Jaiber Ltda, spécialiste de la production de boîtes pliables et d'étiquettes depuis 1976. Cet article analyse l'amélioration des indices de qualité des impressions offset de l'entreprise grâce à la méthodologie Lean Six Sigma. L'objectif principal de cette méthode est d'appliquer un modèle d'amélioration continue stimulant la productivité en réduisant la variabilité des processus garantissant ainsi une meilleure qualité du produit final. Pour améliorer le processus nous avons collecté et analysé des informations pour ensuite recommander des actions de contrôle et d'amélioration. Des outils de diagnostic de type $5 \mathrm{w}$ (cinq questions), Pareto ou le diagramme de cause à effet ont été utilisés, et qui, après analyse, ont permis de définir les causes des problèmes dans les processus d'impression offset. Ce travail a permis aux ingénieurs industriels de l'université Uniminuto - dont la philosophie institutionnelle est l'amélioration de la productivité des petites et moyennes entreprises - de formuler des recommandations visant l'amélioration continue des processus internes et de la productivité de cette entreprise colombienne via la méthodologie DMAIC.

Mots clés: Lean Six Sigma; investigation opérative; gestion de la qualité; amelioration de la qualité; industrie de l'imprimerie, processus de production. 


\section{Introducción}

En los procesos de mejora de la calidad es importante establecer si los productos cumplen con las especificaciones establecidas por el cliente. Esto hace que investigadores como, por ejemplo, Snee (2010), Guevara y Vargas (2011), Gutiérrez-Pulido (2013), Albliwi, Antony, Abdul Halim Lim y Van der Wiele y (2014), o Gijo, Antony y Sunder (2019), entre otros, busquen mejorar procesos metodológicos de calidad. En el presente artículo se tratan los hallazgos y la metodología con el fin de mejorar la calidad de los productos en procesos de impresión offset en la compañía Gráficas Jaiber Ltda. Esta técnica de impresión de uso común transfiere la imagen entintada en una placa a una mantilla de goma, y luego a la superficie de impresión (Coetzee, 2019). Algunas de sus aplicaciones comunes incluyen periódicos, revistas, folletos, papelería y libros (Kipphan, 2001). Por otra parte, cuando un proceso u operación está fuera de control por falta de parametrización y estandarización de actividades se produce una baja en la productividad, lo cual produce, a su vez, errores e inconformidades en el producto terminado (Al-Aomar, Al-Hamed, Khalifeh y Hasan, 2019). Para las empresas dedicadas a la impresión offset es de imperiosa necesidad analizar, mejorar y controlar características fuera de control como lo son, por ejemplo, la calidad de la tinta, las combinaciones de color, el número de manchas, los errores de texto y las dimensiones, entre otros, así como encontrar lo que se denomina causas asignables a la variabilidad de los parámetros de calidad (Mongomery, 2005).

Otros autores (Thomas, Barton y Chuke-Okafor 2009; Busa, 2004; Antony, Krishan, Culle y Kumar, 2012; Dahlgaard y Dahlgaard-Park, 2006; Mustapha, Abu-Hasan y Muda, 2019) plantean modelos organizacionales y de producción orientados a mejorar la calidad. Sin embargo, a pesar de los numerosos modelos de producción y los algoritmos para la resolución de problemas y la toma de decisiones, no se tiene en cuenta el aspecto más importante de la cadena productiva a nivel corporativo: el factor humano. Los ingenieros implicados en este estudio encontraron que la carencia de cultura organizacional, los problemas de comunicación, la alineación de las metas a nivel directivo, administrativo y operativo, pero, sobre todo, la falta de disposición al cam- 
bio en el método de operación, conllevan al colapso del sistema productivo. El presente artículo enfoca la mejora de los índices de calidad en impresión offset de la compañía desde la metodología Lean Six Sigma y busca como objetivo principal aplicar el modelo de mejora continua que permita aumentar la productividad de la compañía. De esta manera, se ponen en evidencia las principales falencias organizacionales que tienen las compañías colombianas y llevan al fracaso corporativo: la falta de constancia en el propósito, el énfasis en el beneficio a corto plazo, culpabilizar a los empleados y la ejecución de los objetivos solo sobre indicadores o cifras visibles (Deming, 1989).

Este artículo muestra cómo la desarticulación de departamentos en la compañía termina por afectar el ambiente laboral, la calidad de producto terminado y, por consiguiente, la falta de respuesta ante reclamos, quejas - garantías, lo que genera un desprestigio muy difícil de cuantificar. Adicionalmente, a causa de la dinámica de los procesos industriales y en razón a cambios en las características del mercado se hace imprescindible estructurar de nuevo cada una de las operaciones que componen ese proceso.

Una vez analizados los datos y la información se proponen oportunidades de mejora del proceso y se le otorgan a la compañía las recomendaciones y las estrategias que permitan corregir los indicadores de calidad. Por último, el presente artículo está estructurado en el marco de resolución de problemas DMAIC - definir, medir, analizar, mejorar y controlar-. Así mismo, debate los resultados obtenidos a partir de la aplicación de ese proceso de evaluación.

\section{Metodología}

\subsection{Definición de la situación actual del proceso}

En esta fase se incluyen los factores influyentes en el producto final, tales como la maquinaria, el mantenimiento, la mano de obra, el método, la materia prima y los insumos. Se ejecutaron reuniones, charlas y capacitaciones con los colaboradores, se recolectaron ideas e información sobre cómo se podría 
adaptar el Lean Six Sigma socializando los objetivos (Sigh y Rathi, 2019), pues es primordial que toda la organización se encuentre familiarizada con los conceptos y principios de Lean Six Sigma.

\subsection{Medición y recopilación de la información}

La información se recolecta con observaciones directas, encuestas a los colaboradores y capacitaciones realizadas, así como con los históricos de datos de inconformidades en los procesos. Posteriormente, se aplica la herramienta de gestión de calidad 5W (Hart, 1996) -esto es, preguntas básicas para la recopilación de información-y el diagrama de causa y efecto con el fin de estructurar la problemática. En un diagrama de Pareto se determinan cuántas causas asignables a los defectos de impresión representan un porcentaje significativo de ocurrencia. De esta manera, se encuentra que gran parte de estos defectos están asociados a la falta de métodos en el proceso de impresión y a eventos o actividades no estandarizadas.

Se procede con un estudio de tiempos en el área de impresión con el propósito de determinar los tiempos estándar, las holguras y los tiempos ociosos permitidos en cada una de las operaciones a ejecutar.

\subsection{Analizar}

Una vez recolectados los datos del proceso en el área de impresión se procede al análisis estadístico y conceptual de la información, con el objetivo de identificar cómo uno de los principales errores se presenta debido a la no existencia de manuales, hojas de ruta y un flujograma de proceso, de modo que se deja a la libre interpretación la elección y el orden de los pasos a ejecutar por parte del operario. Esto, como se verá en posteriores numerales, se ve reflejado en los incrementos de los tiempos de proceso, los tiempos de ocio irregulares y las actividades innecesarias. 


\subsection{Estrategias de mejora}

Se procede a reformar tanto los manuales de funciones de cada operario como los flujogramas de procesos ya existentes con la ayuda tanto de operarios como de supervisores y gerentes. Se ejecutaron charlas y capacitaciones a todos los colaboradores de Grafícas Jaiber Ltda. con relación al programa de mejora continua Lean Six Sigma. Por último, se le otorga a la gerencia una serie de recomendaciones y estrategias dirigidas a controlar y mejorar el proceso de impresión offset.

\section{Resultados y discusión}

\subsection{Situación actual}

Una vez se responden las cinco preguntas en relación con el proceso de impresión offset, esto es, ¿quién realiza el proceso?, ¿qué causa los errores y los defectos?, ¿cuándo se producen?, ¿dónde se generan?, y ¿por qué se producen fallos? $-5 \mathrm{~W}-$, se agruparon las respuestas dadas por los diferentes colaboradores y se realizó el diagrama de Pareto.

La tabla 1 muestra los resultados producto de la combinación de estas dos técnicas para la definición de problemas. En ella se contabilizó el número de errores en los procesos de impresión y se asignaron las causas de dicho fallo. La figura 1 representa los resultados desde la perspectiva del diagrama de Pareto.

Tanto en la tabla 1 como en la figura 1 se muestra que las causas que representan el $80 \%$ de los errores son:

- Cultura organizacional, ambiente laboral y factor humano: $30 \%$.

- No cumplimiento de los procedimientos y falta de control: $24 \%$.

- No se cumple la planeación de producción: $11 \%$.

- Defectos de tinta, color, manchas, texto, dimensiones, etc.: $10 \%$. 
Tabla 1. Causas encontradas y su frecuencia

\begin{tabular}{|c|c|c|}
\hline \multicolumn{2}{|c|}{ Defectos de impresión } \\
\hline$\#$ & Causa & Frecuencia \\
\hline 1 & Cultura organizacional, ambiente laboral y factor humano. & 52 \\
\hline 2 & No cumplimiento en los procedimientos y falta de control. & 41 \\
\hline 3 & No se cumple planeación de producción. & 19 \\
\hline 4 & Defectos en tinta, color, manchas, texto, dimensiones, etc. & 17 \\
\hline 5 & Falta de mantenimiento en la maquinaria. & 18 \\
\hline 6 & Fallas en preprensa. & 11 \\
\hline 7 & Mala calidad insumos y producto terminado. & 8 \\
\hline 8 & Materiales e insumos. & 5 \\
\hline 9 & Otros. & 2 \\
\hline
\end{tabular}

Fuente. Elaboración propia.

Figura 1. Pareto causas encontradas 5W

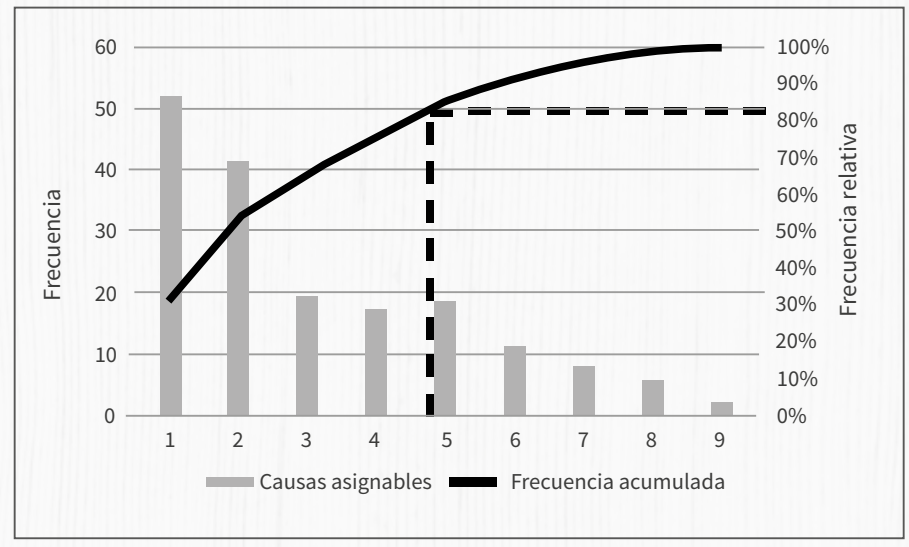

Fuente. Elaboración propia.

En la introducción del presente artículo se hacía referencia a culpabilizar de los errores a los empleados como un error frecuente a nivel corporativo. En este caso, la tendencia es a reconocer los errores a nivel general, pero no asumirlos a nivel individual o personal. 


\subsection{Diagrama de Ishikawa}

Tras realizar una capacitación de Six Sigma White Belt se propuso a los colaboradores identificar causas raíces a los problemas de producción. Esto arrojó como resultado las siguientes causas a resolver:

- diferencias de color respecto al pedido

- vacíos y manchas de impresión

- el material no corresponde a la carta de color

- variación del color

Luego, con base en el concepto "manufactura esbelta», aplicado por Toyota originalmente, se tabularon seis medios tradicionales en el análisis de causas y efectos. La tabla 2 muestra las frecuencias de cada una de las causas encontradas por los colaboradores. Por su parte, la figura 2 representa los resultados desde la perspectiva del diagrama de Pareto.

Tanto en la tabla 2 como en la figura 2 se muestran las causas que representan el $80 \%$ de los errores:

- falta de capacitación hacia el operario: $19 \%$

- falta de mantenimiento y control: $19 \%$

- falta de concientización y responsabilidad del operario: $15 \%$

- calibración de instrumentos: $12 \%$

- no se cumple con el procedimiento: $10 \%$

Tabla 2. Seis emes $-6 \mathrm{M}-$

\begin{tabular}{|c|c|c|c|}
\hline M & $\#$ & Causa & Frecuencia \\
\hline Material & 1,1 & Mala calidad de la tinta. & 6 \\
\hline & 1,2 & Mal estado de las planchas. & 5 \\
\hline Medición & 1,3 & Proveedores. & 3 \\
\hline Método & 3,1 & $\begin{array}{c}\text { Calibración de instrumentos para me- } \\
\text { dir pH, radiómetro y densitómetro. }\end{array}$ & 11 \\
\hline & 3,2 & No se cumple con el procedimiento. & 3 \\
\hline
\end{tabular}




\begin{tabular}{|c|c|c|c|}
\hline Medio ambiente & 4,0 & $\begin{array}{c}\text { Falta de capacitación hacia } \\
\text { el operario. }\end{array}$ & 17 \\
\hline Mano de obra & 5,1 & $\begin{array}{c}\text { Falta de concientización y } \\
\text { responsabilidad del operario. }\end{array}$ & 14 \\
\hline Máquina & 5,1 & Descuido por parte del operario. & 6 \\
\hline
\end{tabular}

Fuente. Elaboración propia.

Figura 2. Pareto causas encontradas seis emes $-6 m-$

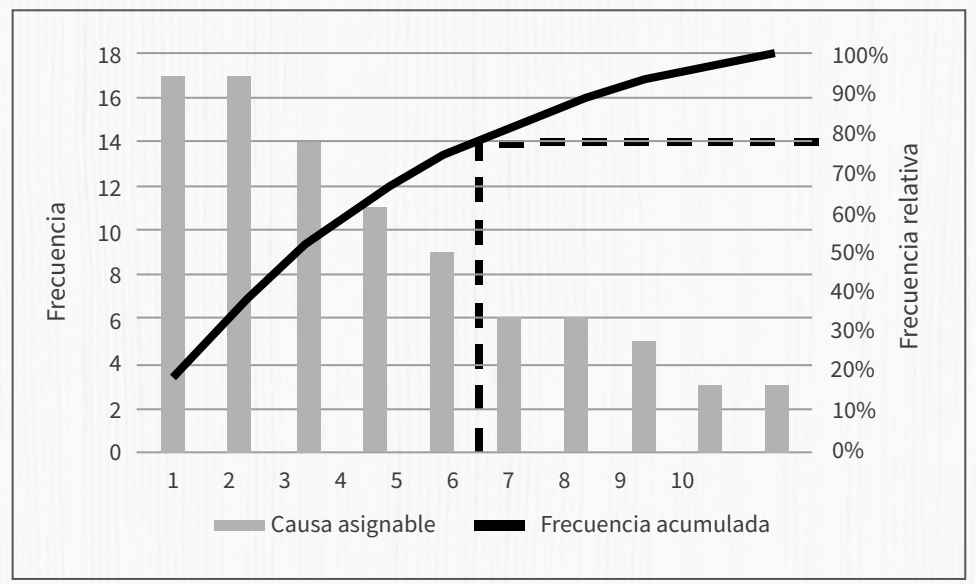

Fuente. Elaboración propia.

\subsection{Tiempos y movimientos}

Con la intensión de conocer la capacidad actual del proceso y lograr comparativos después de implementar la estandarización de cada actividad, se midieron tiempos en las máquinas Speed Master, las cuales están dedicadas a la impresión offset. Las gráficas de las figuras 3, 4, 5 y 6 representan el comportamiento de esos tiempos de impresión en máquina vs $f(x)$, donde $f(x)=\frac{(i-0.5)}{N}, i=$ posición del dato ordenado de menor a mayor y $\mathrm{N}=$ número total de datos. También conocida como la función del papel probabilístico normal, se utiliza en la industria con el fin de evaluar la distribución de los datos (López-Vega, 2010). 
Figura 3. Papel probabilístico normal, tiempos en máquina 1

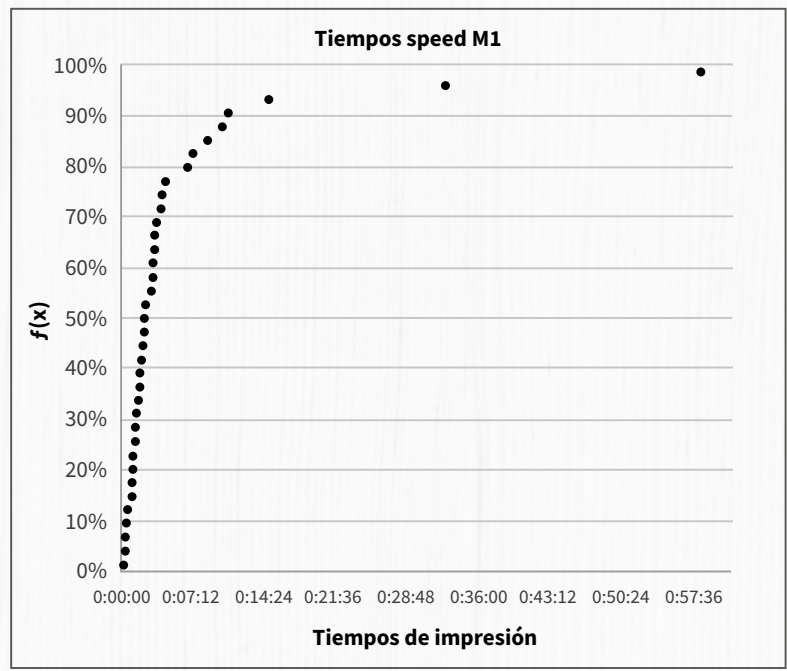

Fuente. Elaboración propia.

De la figura 3 existen dos observaciones que se salen de la tendencia normal de los tiempos de proceso. Esto se debe a tiempos largos para actividades, como lo son:

- El cambio de mantilla en la máquina Speed Master, observando que cuando es necesario realizar esta actividad no se cuenta con las herramientas necesarias. Tiempo: 0:54:44.

- Ajustes de máquina para lograr el balance de la impresión requerida. Tiempo: 0:30:37.

De la figura 4 se deducen tres observaciones que se salen de la tendencia normal de los tiempos de proceso, en razón a los tiempos largos para actividades, como lo son:

- Actividades de transporte de producto terminado a zona de secado. Tiempo: 0:06:46.

- Revisión de los pliegos en medio del proceso. Tiempo: 0:11:02.

- Aplicación de barniz y su revisión. Tiempo: 0:06:36. 
Figura 4. Papel probabilístico normal, tiempos en máquina 2

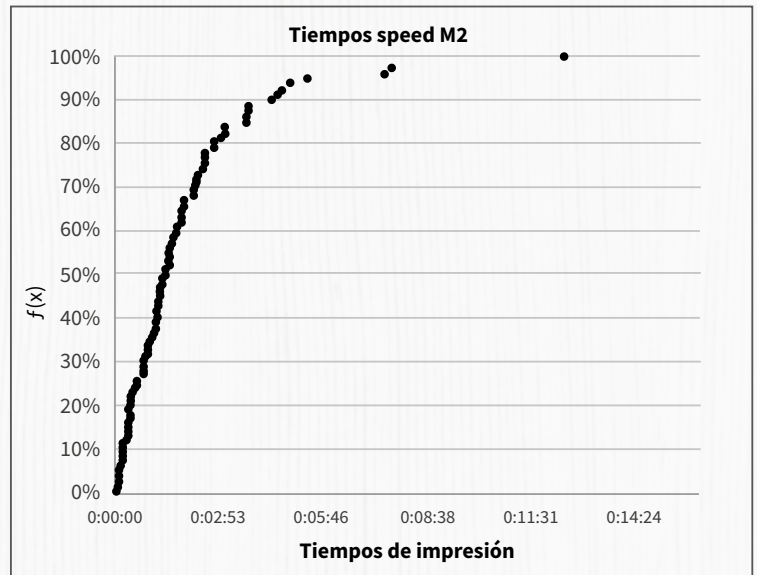

Fuente. Elaboración propia.

En la siguiente toma de tiempos (Figura 5) se presenta una situación común en el sector de la impresión. En este caso, se requiere que el cliente apruebe la impresión para así continuar con el proceso. Esto genera demoras significativas, tal como se refleja en los puntos que se alejan de la tendencia normal de los datos.

Figura 5. Papel probabilístico normal, tiempos en máquina 3

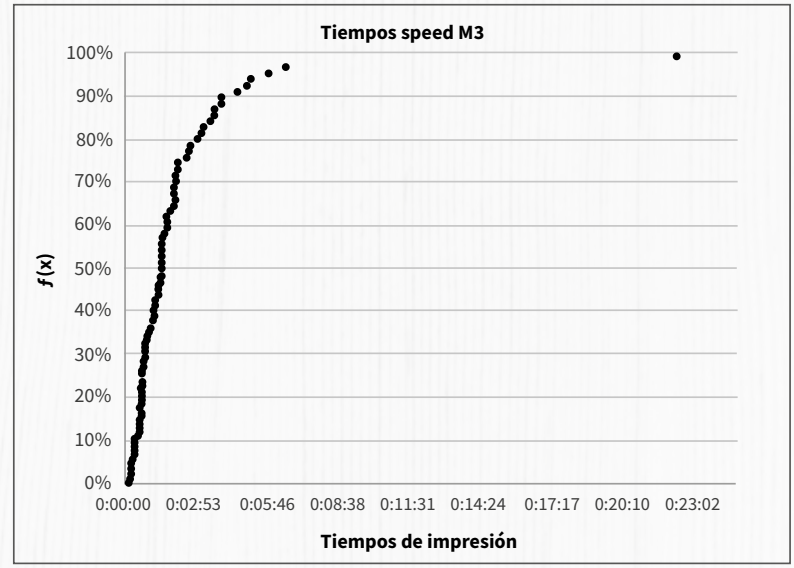

Fuente. Elaboración propia. 
En la figura 5 las observaciones que se salen de la tendencia normal de los tiempos de proceso son:

- Esperar aprobación del cliente. Tiempo: 0:06:07.

- Impresión y confirmación de aprobación del cliente para la orden de trabajo. Tiempo: 0:20:49.

Figura 6. Papel probabilístico normal, tiempos en máquina 4

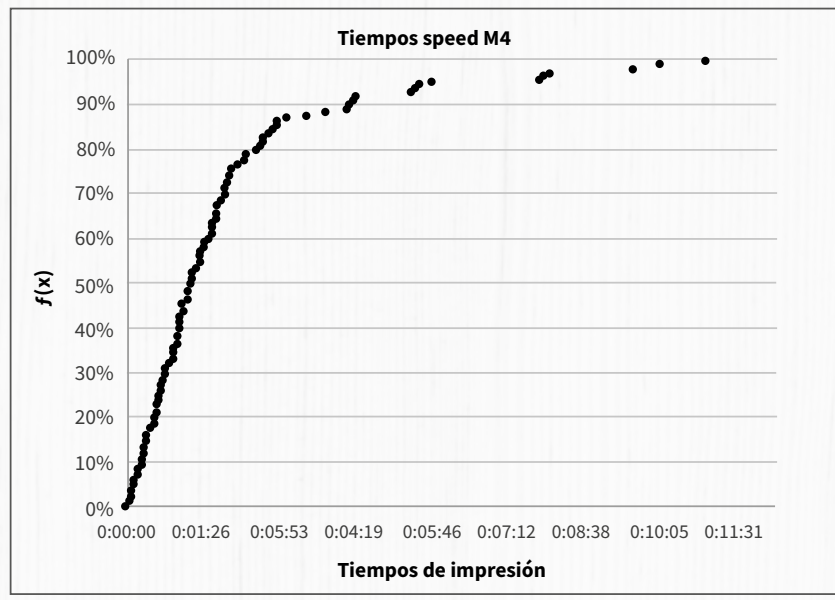

Fuente. Elaboración propia.

Las observaciones que se salen de la tendencia normal en la figura 6 se deben a tiempos largos para actividades, como, por ejemplo:

- Limpieza mantillas-Aux-x4. Tiempo: 0:07:17.

- Desmonte de planchas -Imp y Aux-. Tiempo: 0:07:22.

- Recolección de tinta x2. Tiempo: 0:07:28.

- Impresión maculatura. Tiempo: 0:08:56.

- Organización del material. Tiempo: 0:09:23.

- Limpieza mantillas x4 -Aux-. Tiempo: 0:10:12.

En este caso, se trata de las observaciones que se alejan de la tendencia normalizada, actividades en las que se debe enfocar la compañía con el fin de idear métodos orientados a optimizar sus tiempos. 


\subsection{Eficacia del proceso de impresión Speed Master}

La tabla 3 muestra el cálculo de la eficacia del proceso por máquina, calculado como el cociente del número de tiros -impresiones- por hora y el número de tiros establecidos por hora. Las diferencias evidencian la ineficiencia de los procesos y la subutilización de la maquinaria al no estar trabajando a su capacidad total.

Tabla 3. Eficacias del proceso

\begin{tabular}{|c|c|c|c|c|}
\hline Máquina & $\begin{array}{c}\text { Tiros por } \\
\text { hora }\end{array}$ & $\begin{array}{c}\text { Tiros establecidos } \\
\text { por hora }\end{array}$ & $\begin{array}{c}\text { Diferencia } \\
\text { por hora }\end{array}$ & Eficacia \\
\hline 1 & 2952 & 4400 & 1448 & $67,09 \%$ \\
\hline 2 & 2829 & 4400 & 1571 & $64,30 \%$ \\
\hline 3 & 2607 & 4400 & 1793 & $59,25 \%$ \\
\hline 4 & 1111 & 4400 & 3289 & $25,25 \%$ \\
\hline
\end{tabular}

Fuente. Elaboración propia.

\section{Consideraciones finales y conclusiones}

Con base en los resultados de la tabla 1 la cultura organizacional, el ambiente laboral y el factor humano representan el $30 \%$ de los imperfectos y las demoras en el proceso. Esto lleva a concluir que el aumento significativo de la productividad no solo lo brinda la tecnología de la maquinaria o el tamaño de las instalaciones. Es por esto que la comunicación asertiva y la motivación son imprescindibles a fin de mejorar el proceso y aumentar la productividad. Una vez realizadas las capacitaciones, a la compañía se le recomendó realizar un seguimiento detallado del aprendizaje y del desempeño del personal, con el fin de así realizar comparativos de lo requerido y lo obtenido.

El incumplimiento en los procedimientos y la falta de control tiene una frecuencia importante en los defectos de impresión (véase la Tabla 1). No contar con un procedimiento claro a la hora de ejecutar pedidos, así como la falta de voluntad de las directivas en hacer cumplir el procedimiento, generan 
tiempos de impresión elevados y dificultan, incluso, la interpretación y la definición de las verdaderas actividades. Los ingenieros encontraron que los operarios no tienen una ruta establecida estandarizada, lo cual lleva a que cada uno trabaje con un método propio.

La diferencia de color con respecto a los pedidos tiene que ver no solo con la calidad de los materiales, sino con la definición de las propiedades de las tinturas y el control de variables durante el proceso de impresión.

Los vacíos y las manchas de impresión son superables si se lleva un estricto control documental del proceso, de modo que se podrá evaluar en un posterior estudio cuáles son los factores que influyen en ese fallo. Se recomiendan diseños experimentales como, por ejemplo, las pruebas estadísticas de comparación de poblaciones y el análisis de varianza.

La variación del color y material no correspondiente a la carta de color es una falla operacional y de definición del proceso. Los ingenieros implicados en el proceso de calidad establecieron hojas de ruta y flujogramas claramente definidos entre los trabajadores que influyen de forma directa en el proceso.

El incumplimiento de los procedimientos y la falta de control representa el $24 \%$ de los imperfectos y las demoras del proceso estudiado (véase la Tabla 1). Como se mostró en las gráficas de tiempos en el papel probabilístico normal, se encuentran actividades cuyos tiempos de ejecución difieren significativamente del resto de datos. Estas actividades tienen que ver con costumbres nocivas para estandarizar los tiempos de proceso, como lo son:

- Los desplazamientos no estandarizados. Hacen que los operarios se desplacen por la planta de forma coloquial, lo cual aumenta las demoras en los procesos.

- No hay relevos en las pausas para desayunar, merendar y almorzar. Esto incurre en tener que parar los equipos para estas actividades, lo que supone un posterior consumo de energía adicional para poner de nuevo en marcha el proceso. 
- Distracción de los operarios durante el paro de la máquina por fallos. Esto hace que se desenfoquen del proceso y pierdan ritmo de trabajo, lo que genera conversaciones entre operarios que distraen al resto de las secciones.

Por políticas administrativas el operario tiene que parar el proceso de impresión hasta que el cliente confirme la impresión del siguiente lote. Esto produce tiempos muertos, lo cual lleva a que se incurra en costos de mano de obra improductiva. Los ingenieros concluyeron que esta es la señal más clara de desarticulación entre el departamento administrativo y el de producción. Las decisiones que se toman a nivel administrativo no deben perjudicar las operaciones de producción. Lo anterior es un claro problema de fiabilidad del cliente hacia la compañía y una marcada incertidumbre sobre la calidad de la impresión en el interior de la empresa. Se recomienda mejorar el proceso administrativo en la recepción y la ejecución de los pedidos, ya que las demoras en la confirmación de la impresión son costos que asume la compañía. El proceso debe estar estandarizado y los tiempos para ejecutar un pedido por parte de un cliente deben estar delimitados.

Si se promedian las capacidades calculadas a cada una de las máquinas, tal como se observa en la tabla 3 , el resultado nos lleva a concluir que no se cumple la planeación de producción y su capacidad está en el $53 \%$, debido a los problemas mencionados.

Ahora bien, los defectos de tinta, color, de manchas, de texto, dimensiones, etc., se dan por no definir los tiempos de mantenimiento y calibración de los instrumentos antes y durante el proceso. Por otra parte, la falta de control en factores que pueden afectar la graduación de los colores - como lo son la temperatura y la humedad en el ambiente- no se están controlando. Se recomienda implementar técnicas de control estadístico de procesos en los factores físicos que más afectan los defectos de impresión. 
Toda compañía, sin importar la actividad económica, el sector o el tamaño, debe tener una filosofía empresarial que oriente su proceder en la consecución de sus objetivos. La actuación de cada participante de la compañía tendrá un impacto significativo en los procesos generales de esta. De hecho, las causas asignables a los problemas de la compañía fueron definidas, en su gran mayoría, por los mismos empleados, quienes son conscientes del desorden operacional.

Las filosofías como Lean Six Sigma deben contar con la alineación de objetivos desde la estrategia hacia la táctica. De igual forma, es necesario la constancia de la gerencia en el proceso de cambio de la compañía, pues de lo contrario la filosofía no se puede implementar. Para el caso de Gráficas Jaiber, la gestión de la información y la adaptación a las prácticas en pro de la mejora continua fueron una de las principales dificultades del estudio.

Por último, cabe destacar la principal dificultad -en nuestro caso como ingenieros industriales- de implementar mejoras en la calidad de los procesos. La reticencia al cambio de métodos de producción en los empleados de la planta y la falta de compromiso en hacer cumplir los nuevos métodos por parte de la dirección son un obstáculo para el éxito en la calidad. Confiar netamente en los indicadores visibles, en las certificaciones de calidad, en la automatización y en la nueva maquinaria para garantizar buenos productos es un error de gerencia estratégica. 


\section{Referencias}

Al-Aomar,R.; Al-Hamed, A.; Khalifeh, R.; Hasan, F. (2019). Lean Six Sigma application to improve a water bottling process. Ponencia presentada en la International Conference on Technology Management, Operations and Decisions Marrakech, Marruecos, 21-23 de noviembre. DOI: https://doi. org/10.1109/ITMC.2018.8691151

Albliwi, S.; Antony, J.; Abdul Halim Lim, S.; Van der Wiele, T. (2014). Critical failure factors of lean Six Sigma: a systematic literature review. International Journal of Quality and Reliability Management, 31(9), 10121030. DOI: https://doi.org/10.1108/IJQRM-09-2013-0147

Antony, J.; Krishan, N.; Cullen, D.; Kumar, M. (2012). Lean Six Sigma for higher education institutions (HEIs): challenges, barriers, success factors, tools/techniques. International Journal of Productivity and Performance Management, 61(8), 940-948. DOI: https://doi. org/10.1108/17410401211277165

Busa, R. (2004). Six-Sigma to operational excellence: role of tools and techniques. International Journal of Six Sigma and Competitive Advantage, 1(1), 44-64. DOI: https://doi.org/10.1504/IJSSCA.2004.005277

Coetzee, T. (30 de mayo de 2019). Latest Developments in Printing Technology: What's You Need To Know. Printulu. Recuperado de https://bit.ly/2W22bH4

Dahlgaard, J. J.; Dahlgaard-Park, S. M. (2006). Lean production, six sigma quality, TQM and company culture. TQM Magazine, 18(3), 263-281. DOI: https://doi.org/10.1108/09544780610659998

Deming, W. (1989). Calidad, productividad y competitividad. Madrid: Ediciones Díaz de Santos.

Gijo, E. V.; Antony, J.; Sunder M. V.(2019). Application of Lean Six Sigma in IT support services-a case study. TQM Journal, 31(8), 417-435. DOI: https:// doi.org/10.1108/TQM-11-2018-0168

Guevara. R. D.; J. A., Vargas. (2011). Process capability analysis plot for a product with bilateral specifications. Revista Colombiana de Estadística, 34(2), 287-301. Recuperado de https://bit.ly/2O9DHHO 
Gutiérrez-Pulido, H. (2013). Control estadístico de calidad y Seis Sigma ( $3^{\mathrm{a}}$ ed.). México, D.F: McGraw-Hill.

Hart, G. (1996). The five W's: an old tool for the new task of audience analysis. Technical Communication, 43(2), 139-145. Recuperado de https://bit. ly/2BQU1dU

Kipphan, H. (2001). Handbook of print media: technologies and production methods. Alemania: Springer. DOI: https://doi.org/10.1007/978-3-54029900-4

López-Vega, N. L. (2010). Reducción de demoras en el proceso de impresión en una industria litográfica (Trabajo de grado). Universidad de San Carlos de Guatemala, Guatemala. Recuperado de https://bit.ly/2ZNDXle

Mongomery, D. C. (2005). Introduction to statistical quality control (5a ed.). EE. UU.: Jhon Wiley \& Sons.

Mustapha. M. R.; Abu-Hasan, F.; Muda, M. S. (2019). Lean Six Sigma implementation: multiple case studies in a developing country. International Journal of Lean Six Sigma, 10(1), 523-539. DOI: https://doi. org/10.1108/IJLSS-08-2017-0096

Singh, M.; Rathi, R. (2019). A structured review of Lean Six Sigma in various industrial sectors. International Journal of Lean Six Sigma, 10(2), 622-664. DOI: https://doi.org/10.1108/IJLSS-03-2018-0018

Snee, R. (2010). Lean Six Sigma-getting better all the time. International Journal of Lean Six Sigma, 1(1), 9-29. DOI: https://doi. org/10.1108/20401461011033130

Thomas, A.; Barton, R.; Chuke-Okafor, C. (2009). Applying lean Six Sigma in a small engineering company-a model for change. Journal of Manufacturing Technology Management, 20(1), 113-129. DOI: https://doi. org/10.1108/17410380910925433 